\title{
Is there still a role for cytotoxic chemotherapy after targeted therapy and immunotherapy in metastatic melanoma? A case report and literature review
}

\author{
Aurélien Simon, Hampig Raphael Kourie* and Joseph Kerger
}

\begin{abstract}
Metastatic melanoma has long been considered to have a very poor prognosis and to be chemo-resistant. However, a subgroup of patients with metastatic melanoma presents remarkable responses to chemotherapeutic agents, even in the absence of a response to modern targeted therapies and immunotherapies; accordingly, determining predictive biomarkers of the response to chemotherapies for metastatic melanoma remains a priority to guide treatment in these patients. We report a case study of a patient with B-Raf proto-oncogene serine/threonine kinase-mutated metastatic melanoma harbouring many genetic mutations. The patient did not respond to prior targeted therapies or immunotherapies but experienced a dramatic objective radiological and clinical response to subsequent dacarbazine-based chemotherapy. In the era of targeted therapies and immunotherapies for metastatic melanoma, cytotoxic chemotherapies may still represent an interesting therapeutic weapon in a well-defined subgroup of patients presenting with specific genetic and molecular features.
\end{abstract}

Keywords: Metastatic melanoma, Chemotherapy, Immunotherapy, Checkpoint inhibitors, Vemurafenib, ATM mutation, Chemosensitivity

\section{Background}

Malignant melanoma is a malignancy with a fast growing incidence [1, 2]. Metastatic melanoma has long been considered to exhibit a dismal prognosis and to be chemo-resistant.

In the recent era of emergent targeted therapies and immunotherapies, metastatic melanoma is the first solid tumor to benefit from this therapeutic revolution and has become the pioneer malignancy in these therapeutic areas. The presence of the B-Raf proto-oncogene serine/ threonine kinase $(B R A F)$ V600 mutation in $40 \%-50 \%$ of melanomas and its role as a predictive factor of response to BRAF inhibitors in combination with mitogen-activated protein kinase kinase (MEK) inhibitors were crucial in establishing an appropriate therapeutic management algorithm for metastatic melanomas [3].

\footnotetext{
*Correspondence: Hampig.kourie@hotmail.com
}

Jules Bordet Institute, Free University of Brussels, Brussels, Belgium
Although melanoma has long been considered to be chemo-resistant, cytotoxic chemotherapy represented the only available therapeutic option for metastatic melanoma before the era of targeted therapies and immunotherapies. Many chemotherapy regimens only induced modest response rates; the most common regimens were dacarbazine-based and induced objective response rates (ORRs) ranging from $15 \%$ to $20 \%$ [4]. An observational study has indicated prolonged remission for 7 years [5]. The combination of dacarbazine with other agents, especially cisplatin, produced better results than dacarbazine alone in terms of ORR and progression-free survival but not overall survival [6].

Currently, in BRAF V600-mutated metastatic melanoma, the combination of BRAF and MEK inhibitors is considered the standard of care, with response rates exceeding $70 \%$ for first-line treatment [7]. In $B R A F$ non-mutated metastatic melanoma, immune checkpoint inhibitors have been the standard of care since the 
approval of ipilimumab in March 2011 [8], pembrolizumab in September 2014 [9], and nivolumab in December 2014 as first-line therapies [10]. More recently, the combination of nivolumab and ipilimumab (October 2015) has shown an ORR exceeding $75 \%$, a gain accompanied by higher and more pronounced toxicities than those observed in single-agent immunotherapy trials [11].

In this paper, we report a case of a patient with $B R A F$ mutated metastatic melanoma harbouring many genetic mutations who did not respond to targeted therapies (BRAF and MEK inhibitors) or to immune checkpoint inhibitors, such as ipilimumab and nivolumab, but presented an impressive and dramatic response to subsequent cytotoxic chemotherapy consisting of dacarbazine and cisplatin. We also discuss the potential role of chemotherapy after BRAF and MEK inhibitor treatment and immunotherapy as well as the potential interest and benefit of chemotherapy in particular subgroups of patients.

\section{Case report}

A 56-year-old man with a history of hypercholesterolemia and myocardial infarction presented in December 2013 with a dermatologic lesion in the left lumbar region. The pathologic examination of the excisional biopsy revealed an ulcerated malignant melanoma of $6.5 \mathrm{~mm}$ in thickness (Breslow). The type was a superficial spreading melanoma, and the Clark level was 4.

After a wide excision of the lesion with $2 \mathrm{~cm}$ margins, the pathologic results of the sentinel lymph nodes showed an invasion of malignant melanoma, requiring a subsequent complete left inguinal lymph node dissection. The pathologic TNM stage was pT4bpN1acM0 according to the 7th edition of the American Joint Committee on Cancer/Union for International Cancer Control (AJCC/ UICC) staging system. The primary tumor exhibited the typical BRAF V600E mutation.

Four months later, in April 2014, the patient presented a locoregional cutaneous and subcutaneous relapse in the lumbar region. First-line treatment consisted of the single-agent BRAF inhibitor vemurafenib, which had to be stopped, despite a clinical response, due to unacceptable toxicities, such as a grade 4 skin rash and a grade 2 daily fever. A shift to dabrafenib in combination with trametinib in a medical need programme was initiated in July 2014 and stopped in December 2014 after clinical progression of the lumbar local relapse and of multiple in-transit metastases.

Between January and March 2015, the patient received 4 injections of ipilimumab, a monoclonal anti-cytotoxic T-lymphocyte-associated protein 4 (CTLA4) antibody. The main adverse effect after the fourth injection was excessive fatigue, which was attributed to auto-immune hypophysitis with adrenal and gonadal insufficiencies requiring hormonal substitution of hydrocortisone and topic testosterone, respectively. After 4 doses of ipilimumab, positron emission tomography/computed tomography (PET/CT) unfortunately showed progressive disease and the appearance of lung and lymph node metastases.

Starting in July 2015, the patient was treated with nivolumab (twice every week), a monoclonal antiprogrammed cell death 1 (PD-1) antibody, within the framework of a phase II trial. A CT scan performed after 8 weeks of nivolumab treatment demonstrated clear disease progression, including cutaneous and subcutaneous, lymph node, pleuro-pulmonary, renal, and peritoneal metastases (Fig. 1a, b). At this point, biological analyses indicated elevated serum lactate dehydrogenase (LDH) levels.

Two molecular analyses of the tumor, one using OncoDeep (OncoDNA, Gosselies, Belgium) and the other using the TruSeq Illumina Cancer Panel (Illumina Inc., San Diego, CA, USA), were performed after the failure of nivolumab (at the end of August 2015). The results were discordant: the OncoDNA detected only one BRAF V600E mutation, whereas the Illumina Panel (TruSeq Amplicon Cancer Panel) detected $B R A F$ V600E-F-box and WD repeat domain containing 7 R385C mutations (FBXW7), a kinase domain insert receptor $\mathrm{Q} 472 \mathrm{H}$ variant $(K D R)$, a V-Ki-ras2 Kirsten rat sarcoma viral oncogene homologue G12D mutation (KRAS), a tumor protein P53 P72R variant (P53), and a polymorphism of Ataxia telangiectasia mutated (ATM) - c. $8850+60 \mathrm{~A}>\mathrm{G}$.

Since September 2015, the patient had received 4 cycles of cytotoxic chemotherapy consisting of intravenous injections of dacarbazine $\left(350 \mathrm{mg} / \mathrm{m}^{2}\right)$ and cisplatin $\left(25 \mathrm{mg} / \mathrm{m}^{2}\right)$ for 3 consecutive days, given every 3-4 weeks. An ongoing, impressive, and dramatic response of all metastases (the sizes decreased by more than $80 \%$ ) was documented after 3 cycles of chemotherapy (Fig. 1c, d).

During chemotherapy, a second biopsy was performed, and the same mutations were detected, but there was a difference in the percentage of cells with the BRAF V600E mutation (41\% in August 2015 and 36\% in November 2015).

After the failure of checkpoint inhibitors, an immunological biomarker and microenvironment analysis revealed the absence of $\mathrm{PD}-1 /$ programmed death-ligand 1 (PD-L1) (Ventana biomarker assay) staining, the absence of CD20 (B cells) staining, and diffuse and weak CD3 (T cells) staining.

We summarized the treatment provided to this patient in a flow chart (Fig. 2). 

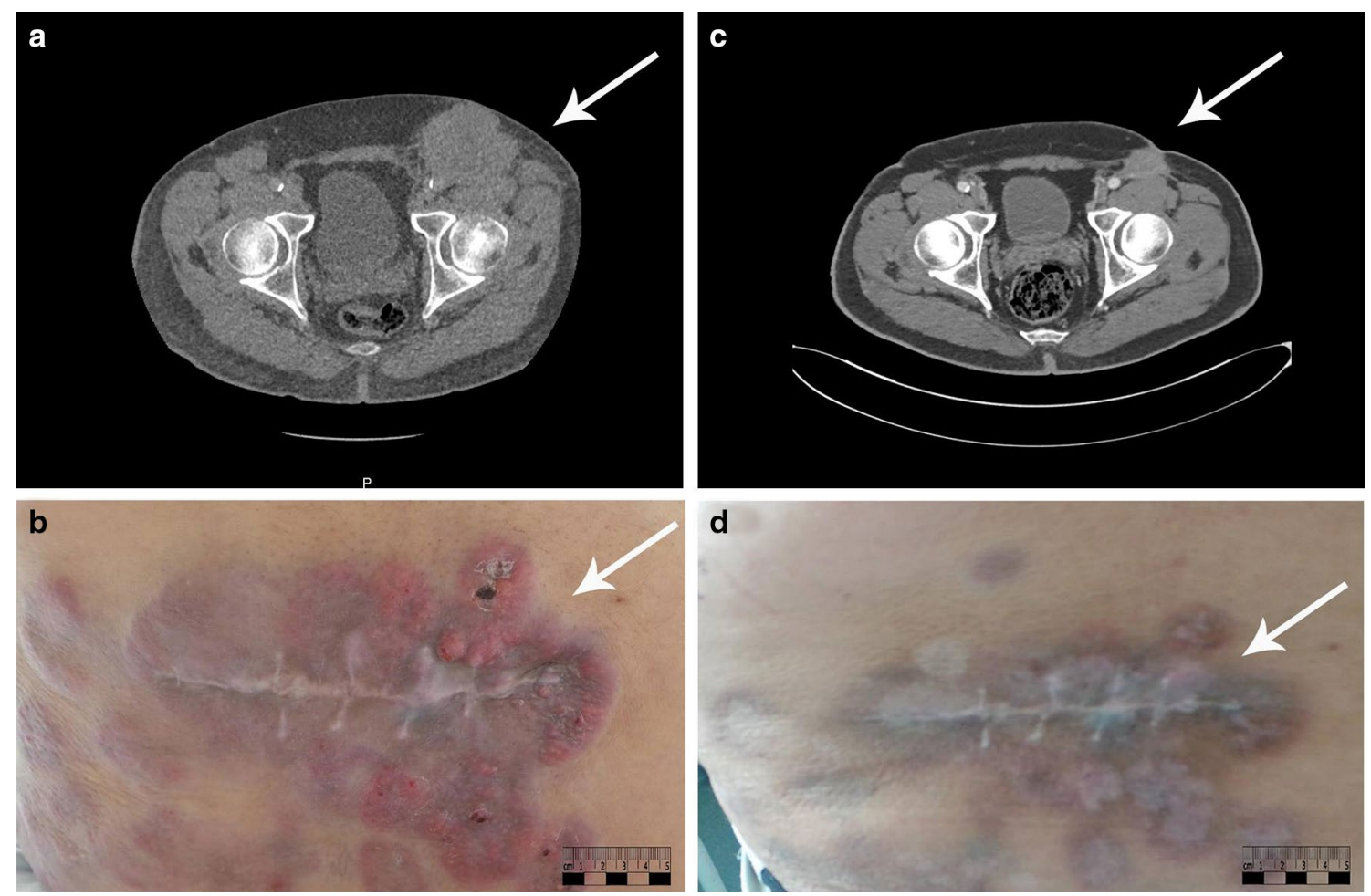

Fig. 1 Computed tomography $(\mathrm{CT})$ and macroscopic images of the inguinal lesion before and after 3 cycles of chemotherapy. a CT scan shows a subcutaneous metastatic melanoma lesion (arrow) of $76 \mathrm{~mm} \times 63 \mathrm{~mm}$ in the left inguinal area before chemotherapy. b Cutaneous metastatic melanoma lesions (arrow) were nodular and inflammatory before chemotherapy. c CT scan shows that the size of the subcutaneous metastatic melanoma lesion (arrow) decreased to $31 \mathrm{~mm} \times 35 \mathrm{~mm}$, with a reduction of 48\%, after 3 cycles of chemotherapy. d Cutaneous metastatic melanoma lesions (arrow) exhibited massive shrinkage, leaving a fibrotic quality of the skin, after 3 cycles of chemotherapy

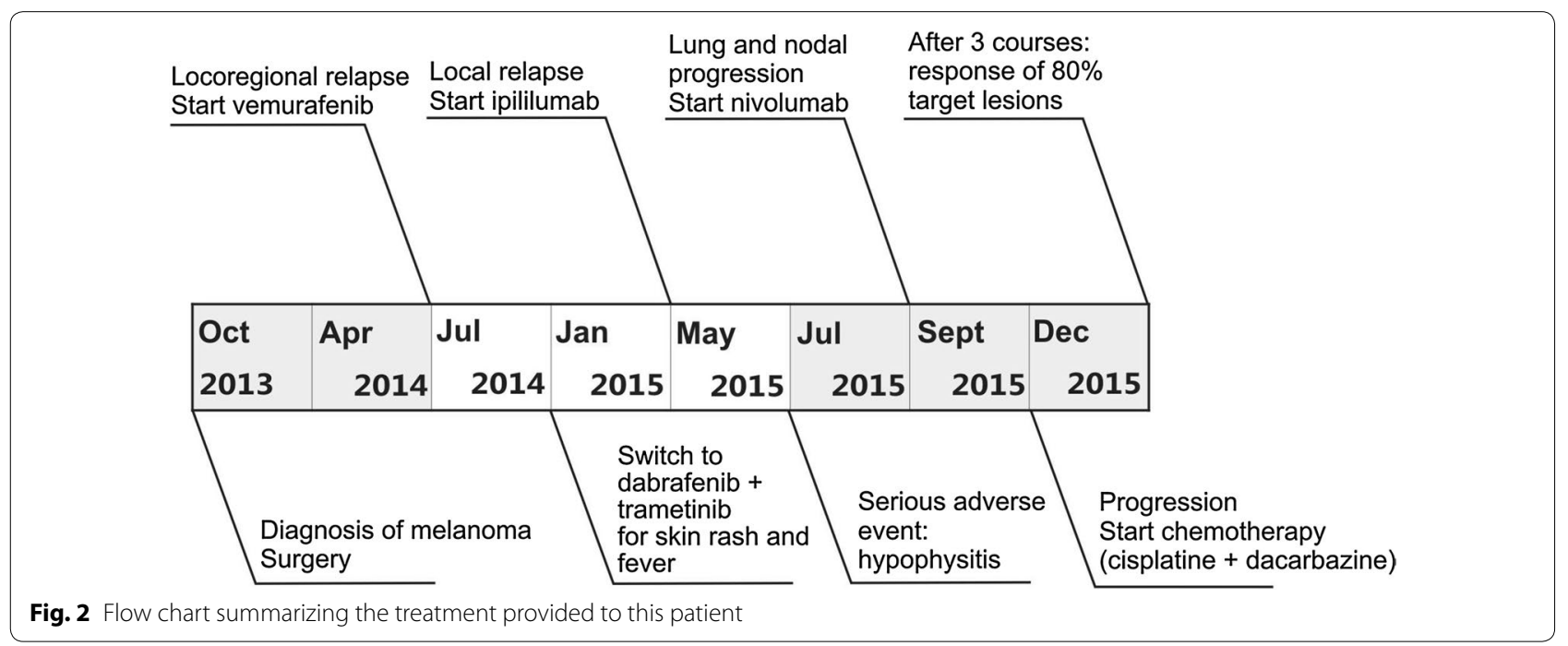

\section{Discussion}

The particular clinical feature of our case was the presence of multiple genetic mutations in the tumor, which did not respond to targeted therapies or checkpoint inhibitors but exhibited a major response to dacarbazine and cisplatin combination chemotherapy in fifth-line therapy.

Apart from the differences (e.g., depth of coverage, number of genes analyzed, and devices and analysis systems) between the OncoDeep test and Illumina panel, 
the discordant results (i.e., the greater number of mutations detected using the Illumina panel) may be explained by tumor heterogeneity due to the different origins of the two samples.

This rare case raises a number of questions. Is there a subgroup of metastatic melanomas that still benefit from cytotoxic chemotherapy? Are there any predictive factors leading to this response? Should the presence of the observed genetic mutations in metastatic melanoma be considered a predictive factor for chemo-sensitivity? Is there a potential role for immune checkpoint inhibitors that render these tumors more chemo-sensitive by modifying the microenvironment?

Many hypotheses can be considered with respect to these questions. The first and strongest hypothesis is that the observed response is explained by the presence of an ATM mutation in this tumor. The ATM gene is responsible for the repair of DNA double-strand breaks [12]. The presence of an ATM mutation leads to a dysfunction in the repair process for DNA double-strand breaks and consequently could render the tumor more chemosensitive, especially to platinum agents, according to the literature $[13,14]$. This process is comparable to breast cancer 1 gene (BRCA1)-mutated breast cancer, which exhibits acceptable sensitivity to platinum agents and/ or poly(ADP-ribose) polymerase (PARP) inhibitors. By extrapolation, the use of PARP inhibitors could be considered an interesting therapeutic modality in the progression of chemotherapy.

A second hypothesis may be the "terra incognita" effect of immunotherapy (anti-CTLA4 and anti-PD-1) on the subsequent response to chemotherapy. Cytotoxic agents seem to enhance the anti-tumor immune response by releasing antigens after cellular destruction [15, 16]. Some basic researches on immunological biomarkers and microenvironments, e.g., studies of intratumoral lymphoid infiltrates with intratumoral PD-L1 expression and the interferon-gamma pathway in tumor tissue, show that these factors can predict the response to immune checkpoint inhibitors $[17,18]$. In fact, high expression of PD-L1 and the presence of tumor-infiltrating lymphocytes are associated with better responses to checkpoint inhibitors $[19,20]$.

Finally, to answer these questions, a retrospective mutation-based study could be used to evaluate the response rate of metastatic melanomas to different therapeutic modalities according to each individual mutation. Based on the results of this observational study, a randomized trial aimed at comparing chemotherapy to targeted therapies and checkpoint inhibitors based on different mutation profiles should be launched. A similar methodology will be used to confirm or clarify the sustained role of chemotherapy in well-defined subgroups of patients, despite the encouraging and promising results of targeted therapies and/or immunotherapies.

\section{Conclusions}

We are currently in an exciting era of promising new treatment options for malignant melanoma. Cytotoxic chemotherapy (especially dacarbazine and cisplatin) could nevertheless remain an invaluable therapeutic weapon in specific cases with chemosensitizing mutations.

\section{Authors' contributions}

SA and KHR initiated the review; SA and KHRS performed the review, wrote the paper, and analysed the data. All authors read and approved the final manuscript.

\section{Acknowledgements \\ None.}

Competing interests

The authors declare that they have no competing interests.

Received: 16 February 2016 Accepted: 30 September 2016

Published online: 13 January 2017

References

1. Erdmann F, Lortet-Tieulent J, Schüz J, Zeeb H, Greinert R, et al. International trends in the incidence of malignant melanoma 19532008-are recent generations at higher or lower risk? Int J Cancer. 2013:132:385-400.

2. Boyle P, Doré JF, Autier P, Ringborg U. Cancer of the skin: a forgotten problem in Europe. Ann Oncol. 2004;15:5-6.

3. Long GV, Stroyakovskiy D, Gogas H, Levchenko E, de Braud F, Larkin J, et al. Dabrafenib and trametinib versus dabrafenib and placebo for Val600 BRAF-mutant melanoma: a multicentre, double-blind, phase 3 randomised controlled trial. Lancet. 2015:386:444-51.

4. Bhatia S, Tykodi SS, Thompson JA. Treatment of metastatic melanoma: an overview. Oncology (Williston Park). 2009;23:488-96.

5. Ahmann DL, Creagan ET, Hahn RG, Edmonson JH, Bisel HF, Schaid DJ. Complete responses and long-term survivals after systemic chemotherapy for patients with advanced malignant melanoma. Cancer. 1989;63:224-7.

6. Lui P, Cashin R, Machado M, Hemels M, Corey-Lisle PK, Einarson TR. Treatments for metastatic melanoma: synthesis of evidence from randomized trials. Cancer Treat Rev. 2007:33:665-80.

7. Long GV, Stroyakovskiy D, Gogas H, Levchenko E, de Braud F, Larkin J, et al. Combined BRAF and MEK inhibition versus BRAF inhibition alone in melanoma. N Engl J Med. 2014;371:1877-88.

8. Hodi FS, O'Day SJ, McDermott DF, Weber RW, Sosman JA, Haanen JB, et al. Improved survival with ipilimumab in patients with metastatic melanoma. N Engl J Med. 2010;363:711-23.

9. Ribas A, Puzanov I, Dummer R, Schadendorf D, Hamid O, Robert C, et al. Pembrolizumab versus investigator-choice chemotherapy for ipilimumab-refractory melanoma (KEYNOTE-002): a randomised, controlled, phase 2 trial. Lancet. 2015;16:908-18.

10. Robert C, Long GV, Brady B, Dutriaux C, Maio M, Mortier L, et al. Nivolumab in previously untreated melanoma without BRAF mutation. $N$ Engl J Med. 2015:372:320-30.

11. Larkin J, Chiarion-Sileni V, Gonzalez R, Grob JJ, Cowey CL, Lao CD, et al. Combined nivolumab and ipilimumab or monotherapy in untreated melanoma. N Engl J Med. 2015;373:23-34.

12. Lee $\mathrm{J}-\mathrm{H}$, Paull TT. Activation and regulation of ATM kinase activity in response to DNA double-strand breaks. Oncogene. 2007;26:7741-8. 
13. Lavin MF, Kozlov S. ATM activation and DNA damage response. Cell Cycle. 2007;6:931-42.

14. Basu A, Krishnamurthy S, Basu A, Krishnamurthy S. Cellular responses to cisplatin-induced DNA Damage, cellular responses to cisplatin-induced DNA damage. J Nucleic Acids. 2010;2010:201367. doi:10.4061/2010/201367

15. Weir GM, Liwski RS, Mansour M. Immune modulation by chemotherapy or immunotherapy to enhance cancer vaccines. Cancers. 2011;3:3114-42.

16. Bracci L, Schiavoni G, Sistigu A, Belardelli F. Immune-based mechanisms of cytotoxic chemotherapy: implications for the design of novel and rationale-based combined treatments against cancer. Cell Death Differ. 2014;21:15-25.

17. Lee SK, Seo SH, Kim BS, Kim CD, Lee JH, Kang JS, Maeng PJ, Lim JS. IFN- $\gamma$ regulates the expression of $\mathrm{B} 7-\mathrm{H} 1$ in dermal fibroblast cells. J Dermatol. 2005:40:95-103.
18. Gajewski TF, Louahed J, Brichard VG. Gene signature in melanoma associated with clinical activity: a potential clue to unlock cancer immunotherapy. Cancer J. 2010;16:399-403.

19. Loi S, Sirtaine N, Piette F, Salgado R, Viale G, et al. Prognostic and predictive value of tumor-infiltrating lymphocytes in a phase III randomized adjuvant breast cancer trial in node-positive breast cancer comparing the addition of docetaxel to doxorubicin with doxorubicin-based chemotherapy: BIG 02-98. J Clin Oncol. 2013;31:860-7.

20. Remon J, Chaput N, Planchard D. Predictive biomarkers for programmed death-1/programmed death ligand immune checkpoint inhibitors in non-small cell lung cancer. Curr Opin Oncol. 2016;28:122-9.

\section{Submit your next manuscript to BioMed Central and we will help you at every step:}

- We accept pre-submission inquiries

- Our selector tool helps you to find the most relevant journal

- We provide round the clock customer support

- Convenient online submission

- Thorough peer review

- Inclusion in PubMed and all major indexing services

- Maximum visibility for your research

Submit your manuscript at www.biomedcentral com/submit 